\title{
« L'affaire du bypass gastrique en oméga » (improprement appelé « mini-bypass »)
}

\section{J. Dargent $\cdot$ M. Pigeyre $\cdot$ L. Genser}

(C) Lavoisier SAS 2019

\section{Commentaire de la rédaction de la revue Obésité}

À l'occasion de ce qu'il faut bien appeler une affaire sérieuse, le déremboursement du bypass gastrique en oméga, une technique chirurgicale largement pratiquée, la chirurgie bariatrique française vis une séquence particulière de sa jeune histoire. Afin de comprendre le débat actuel, nous avons décidé de publier dans ce numéro deux documents essentiels.

D'une part, le communiqué de presse relatif au rapport émis par la Haute Autorité de santé (HAS) sur l'évaluation du bypass gastrique en oméga. C'est sur la base des conclusions émises dans ce rapport que la Caisse nationale d'Assurance maladie (CNAM) a décidé de suspendre le remboursement de cette intervention dans l'attente d'études complémentaires et d'un niveau de preuve suffisant. Ce rapport est basé sur l'analyse objective de la littérature et reflète l'opinion majoritaire du groupe de travail convoqué par la HAS ${ }^{1}$.

D'autre part, nous publions la réaction du Professeur Jean-Marc Chevallier, chirurgien hospitalier et universitaire, qui a étudié cette intervention de longue date, a publié de nombreux travaux sur ce sujet, et qui désapprouve la décision des tutelles².

La position de cet universitaire peut être discutée, mais il nous semble important de la relayer sachant bien qu'elle n'engage pas la rédaction de la revue. La décision des tutelles s'impose aux acteurs de la chirurgie de l'obésité en France, et à notre société savante, la SOFFCO-MM, qu'elle soit regrettable ou non. Souhaitons tout de même que le débat puisse se poursuivre dans une atmosphère sereine !

J. Dargent $(\bowtie)$

Polyclinique Lyon Nord, 65 rue des Contamines, 69140 Rillieux-la-Pape, France

e-mail : jerome.dargent@polyclinique-rillieux.fr

\section{Pigeyre}

Service de nutrition, Centre hospitalo-universitaire de Lille, 59037 Lille Cedex

\section{Genser}

AP-HP, Service de chirurgie digestive et métabolique,

Hôpital Avicenne, AP-HP, Centre intégré Nord francilien de l'obésité, 125 rue de Stalingrad, 93000 Bobigny.

Université Paris XIII-UFR SMBH « Léonard de Vinci », France

1. Rapport disponible sur internet: https://www.has-sante.fr/upload/ docs/application/pdf/2019-09/rapport_bypass_gastrique_en_omega_ vd.pdf.

2. Il faut se référer au rapport de la HAS pour les détails auxquels il est fait référence dans cet article. 


\section{Communiqué de presse de la HAS : La HAS est défavorable au remboursement du bypass gastrique en oméga ${ }^{1}$}

Les techniques de bypass sont fréquemment utilisées dans la chirurgie de l'obésité en France. Mais si le bypass dit en Y a été évalué et validé, le bypass dit en oméga ne l'avait encore jamais été. Or cette pratique s'est diffusée largement en France, car elle serait plus simple à réaliser pour les chirurgiens. Au terme de son évaluation, la HAS préconise de ne plus y avoir recours lorsque l'anse fait $200 \mathrm{~cm}$ ou plus ${ }^{2}$ et est défavorable à son remboursement dans le traitement chirurgical de l'obésité en raison de données insuffisantes pour établir son efficacité et de l'existence de potentielles complications graves associées.

La chirurgie bariatrique est une option pour les personnes atteintes d'obésité sévère ou massive en échec thérapeutique. Chaque année, plus de 50000 personnes sont opérées en France. Parmi les techniques chirurgicales, les plus utilisées sont la sleeve (32000 par an) et les bypass (13 000 par an). Les bypass consistent en une réduction de la taille de l'estomac associée à un court-circuitage d'une partie de l'intestin grêle. Ce court-circuit a d'abord été réalisé « en Y », technique évaluée et recommandée par la Haute Autorité de santé ${ }^{3}$, puis une autre technique - dite « en oméga »-s'est largement diffusée.

La HAS a évalué le bypass en oméga, jusque-là objet d'un programme hospitalier de recherche clinique (PHRC) dont les résultats viennent d'être publiés, et statue sur deux questions : peut-il être une solution au bypass en $\mathrm{Y}$ et convient-il de l'admettre au remboursement?

\section{Ne plus réaliser de bypass en oméga avec une anse à $200 \mathrm{~cm}$}

La technique du bypass en oméga a été développée initialement pour pallier la complexité de l'opération du bypass en Y. Plus simple à réaliser, elle est depuis quelques années largement pratiquée en France, le remboursement étant rendu possible par le caractère générique du libellé de l'acte remboursé par l'Assurance maladie ( bypass », sans précision).

Sur la base des données publiées, notamment des résultats du PHRC, et de la position d'un groupe de professionnels, la HAS conclut aujourd'hui que cette technique ne représente pas une alternative pertinente au bypass en $\mathrm{Y}$ et préconise qu'elle ne soit pas remboursée par l'Assurance maladie. En effet, les études révèlent un plus grand nombre de complications graves - parmi lesquelles des carences

1. Le communiqué de presse de l'HAS a été publié ce 23 sept. 2019. Date de mise en ligne : 23 sept. 2019 sur le site de la SOFFCO-MM.

2. C'est-à-dire que la dérivation exclut $200 \mathrm{~cm}$ du tube digestif.

3. Recommandation de bonnes pratiques «Obésité : prise en charge chirurgicale chez l'adulte », 2009. sévères, notamment en vitamines et minéraux, des reflux biliaires... De surcroit, il n'existe pas de données probantes sur une meilleure efficacité du bypass en oméga par rapport au bypass en $\mathrm{Y}$.

Les données disponibles concernent le bypass en oméga avec une anse de $200 \mathrm{~cm}$. Les données publiées manquent sur le bypass en oméga réalisé avec une anse de $150 \mathrm{~cm}$, une modalité alternative qui limiterait les complications nutritionnelles. Afin de pouvoir se prononcer, la HAS préconise de les réaliser dans le cadre d'essais cliniques uniquement.

Concernant les patients déjà opérés, la HAS recommande qu'ils bénéficient du même suivi que ceux opérés selon la technique du bypass en $\mathrm{Y}$ mais avec une vigilance particulière sur la survenue de complications nutritionnelles et liées au reflux biliaire - dont on ignore les conséquences à long terme. La HAS recommande en particulier une fibroscopie cinq ans après l'intervention chirurgicale.

\section{Vers une chirurgie de l'obésité plus sûre et plus pertinente}

La HAS a programmé d'autres travaux pour évaluer la pertinence, l'efficacité et la sécurité des techniques de chirurgie bariatrique autres que les quatre aujourd'hui recommandées (l'anneau gastrique, la sleeve, le bypass en $Y$ et la dérivation bilio-pancréatique). Elle va ainsi réaliser un état des lieux des techniques de chirurgie bariatrique actuellement en développement. L'enjeu est de les évaluer avant qu'elles ne se diffusent dans la pratique courante.

Par ailleurs, dans le cadre de ses travaux en matière d'amélioration de la qualité et de la sécurité des soins, la HAS développe et recueille des indicateurs spécifiques à la chirurgie bariatrique et va produire des documents sur le parcours obésité et surpoids : indications des interventions de chirurgie bariatrique, évaluation préopératoire, suivi postopératoire.

La HAS rappelle que la chirurgie est un traitement de seconde intention de l'obésité - c'est-à-dire après échec d'un traitement médical, nutritionnel, diététique et psychothérapeutique bien conduit pendant 6-12 mois - et concerne les patients dont l'indice de masse corporelle (IMC) est supérieur ou égal à $40 \mathrm{~kg} / \mathrm{m}^{2}$, ou dont l'IMC est supérieur ou égal à $35 \mathrm{~kg} / \mathrm{m}^{2}$ associé à au moins une comorbidité susceptible d'être améliorée après la chirurgie. En cas de réussite, elle aide à une perte de poids importante et persistante. Pourtant cet acte lourd ne doit être réalisé qu'à l'issue d'une décision médicale partagée, avec une information claire sur les techniques existantes, leurs avantages et inconvénients, leurs conséquences, les complications et sur la nécessité d'un suivi médical tout au long de la vie. 


\section{Réaction du Pr Jean-Marc Chevallier ${ }^{1}$ Quand la gestion des conflits d'intérêt l'emporte sur l'expérience}

\section{Le contexte}

Alertée par certains chirurgiens qui ont envoyé des signaux de sécurité graves, la HAS a publié un rapport sur $L e$ traitement chirurgical de l'obésité sévère et massive par court-circuit gastro-jéjunal avec anse en oméga.

Ce rapport a été rédigé selon les règles de la HAS, après analyse de la littérature et une réunion d'experts qui s'est tenue le 11 juin 2019.

Il est déclaré : l'objectif principal de l'évaluation est de définir si la technique BPGO peut remplacer dans tout ou partie de ses indications le $B P G Y$.

Le choix de comparer le bypass en oméga (dont la dénomination officielle reconnue par l'IFSO, doit être OAGB One anastomosis Gastric Bypass) au bypass en Y (RYGB) témoigne d'une méconnaissance du problème. L'OAGB est une technique plus hypo-absorptive qu'il faut plutôt ranger dans les dérivations bilio-pancréatiques et donc ne pas comparer au RYGB. C'est d'ailleurs tout le problème.

La conclusion en a été : la HAS considère que le bypass gastrique en oméga (BPGO) réalisé avec une anse biliopancréatique (BP) à $200 \mathrm{~cm}$ (ou plus longue) ne constitue pas, dans le traitement chirurgical de l'obésité massive et sévère (avec comorbidité), une technique validée; elle n'est donc pas une alternative au bypass gastrique de Roux-en-Y (BPGY).

Cette conclusion a entraîné un déremboursement du OAGB par la CNAM, ce qui conduit à interdire la troisième opération bariatrique la plus réalisée au monde après le bypass en Y et la sleeve gastrectomie. L'OAGB est devenue l'opération la plus réalisée en Inde et en Israël par exemple.

Pratiquant la chirurgie de l'obésité depuis près de 30 ans, j'ai donc participé aux débuts de la lutte contre ce fléau. J'ai présidé le Congrès mondial de l'IFSO à Paris en 2009, ai été membre fondateur, secrétaire général puis président de la SOFFCO-MM. Travaillant dans un hôpital universitaire (HEGP, Paris) je me suis obligé dès le début à publier nos résultats dans les revues de référence internationale, d'abord sur l'anneau gastrique puis sur le bypass. J'ai commencé à étudier l'OAGB en 2006, après la première étude randomisée de WJ Lee. Je me suis astreint à étudier cette opération de la façon la plus académique en publiant toutes les complications parmi les 1000 premiers patients [1], les résultats fonctionnels à 5 ans [2] et les rémissions des 100 premiers

\section{J.M. Chevallier}

Membre de l'Executive Board de l'IFSO, president-Elect de l'IFSO-EC, past-président de la SOFFCO-MM ;

Service de Chirurgie Digestive, Hôpital européen Georges

Pompidou, 20 rue Leblanc, 75015 Paris.

e-mail : Jean-marc.chevallier@aphp.fr diabètes opérés [3]. Connaissant la controverse autour des risques potentiels de l'OAGB, j'avais spécifié que s'il y avait la moindre raison de récuser cette opération je l'écrirais. Treize ans plus tard, je suis convaincu que cette opération est plus efficace et moins morbide que le bypass en $\mathrm{Y}$ et surtout que la sleeve gastrectomie. Cette conclusion rejoint celles émises par ceux qui ont une longue expérience de chirurgie de l'obésité dans le monde : l'an dernier l'IFSO (Fédération internationale de chirurgie de l'obésité) a désigné un groupe de travail qui a repris tous les travaux de la littérature et a pris position en faveur de cette opération [4]. Cette année j'ai co-présidé, avec le président de l'IFSO le Pr Almino Ramos, une réunion de consensus international qui s'est tenue les 18 et 19 juillet 2019 à Hamburg (Allemagne) : 52 experts du monde entier (14 venus d'Europe, 9 d'Amérique du Sud, 10 du Moyen-Orient-Afrique, 11 d'Asie-Pacifique et 8 d'Amérique du Nord) ont répondu à plus de 80 questions débouchant sur un consensus favorable à l'OAGB et la publication d'un livre [5] présenté au $24^{\mathrm{e}}$ congrès mondial de l'IFSO qui s'est tenue à Madrid du 4 au 7 septembre dernier.

Il y a donc un consensus mondial pour développer cette intervention, et la France pourrait se marginaliser en l'interdisant, ce serait contre-productif.

\section{Le rapport}

Pour des raisons de conflit d'intérêt, ma candidature à la réunion d'expertise de la HAS n'a pas été prise en compte et je n'ai pas eu connaissance de ce rapport avant sa publication. Or le rapport de la HAS n'est pas objectif.

Voici les principales erreurs que j'ai relevées dans ce rapport :

- Page 8. Il n'a jamais été question de remplacer le bypass en Y par l'OAGB. Le RYGB est restrictif alors qu'OAGB est plutôt hypo-absorptif. Il s'agit de déterminer dans quel cas faire l'un ou l'autre.

- Pages 6/32/37/61. A chacune de ces pages apparaît le risque de cancer de l'œsophage. Cela a été pour nous une priorité dès le début. Cliniquement le reflux biliaire invalidant ne touche que $1 \%$ des patients, mais afin d'obtenir une réponse rapide à ce risque potentiel nous avons fait des travaux expérimentaux chez le rat, lesquels ne sont pas référencés dans ce rapport. Nous avons gardé ces rats en vie 16 semaines (ce qui équivaut à 16 ans d'une vie humaine), nous n'avons trouvé aucun cancer, ni dysplasie et surtout aucune augmentation des gènes spécifiques marqueurs de EBO et du cancer œeso-gastrique [6]. Il existe maintenant quatre articles préliminaires qui ont conduit à une thèse de doctorat soutenue par un de nos élèves [7]. 
- Page 12. Il est faux d'écrire que «des auteurs suggéraient dès 2008 une longueur 'personnalisée' en fonction de l'IMC du patient. Cependant, cette stratégie ne semble pas optimale et exposerait à des complications nutritionnelles et hépatiques ». C'est l'inverse : le travail de Carbajo qui personnalise en fonction de la longueur du grêle et de l'IMC sur 1200 patients suivis plus de 10 ans ne rapporte aucun patient réopéré pour malnutrition [8].

- Page 14. Atteinte hépatique, avec impact négatif sur les enzymes hépatiques (p. 31), pouvant entraîner la décision de réversion de l'opération (retour à une anatomie normale). Non, l'élévation des transaminases se normalise après la première année [9].

- Page 24. Dans ce rapport seuls trois essais randomisés (RCT) sont cités. Il en existe au moins sept. Outre ceux de WJ Lee, Robert, et Ruiz-Tovar, je rappellerai :

- Seetharamaiah S, Tantia O, Goyal G et al (2017) LSG vs OAGB- 1 year follow up Data - a Randomized Control Trial. Obes Surg; 27:948-54

- Lee WJ, Chong K, Ser KH et al. (2011) Gastric bypass vs Sleeve gastrectomy for Type 2 Diabetes Mellitus (2011). A randomized control trial. Arch Surg 146 (2):143-8

- Lee WJ, Chong K, Lin YH et al. (2014) Laparoscopic Sleeve gastrectomy versus Single anastomosis (Mini-) gastric bypass for the treatment of Type 2 Diabetes Mellitus: 5-year results of a randomized Trial and study of Incretin Effect. Obes Surg 24:1552-62

- Darabi S, Taledpour M, Zeinoddini A et al. (2013) Laparoscopic gastric plication versus mini-gastric bypass surgery in the treatment of morbid obesity: a prospective randomized controlled clinical trial. Surg Obes Relat Dis. 9(6):914-9

Même si les RCT non retenues par le rapport concernent surtout le rôle du OAGB sur le diabète, il s'agit de données essentielles. Les sept études randomisées ont été revues dans la méta-analyse qui a conduit au Position Statement en faveur de l'OAGB par l'IFSO [4] et par l'ASMBS [10]. Pourquoi en faire abstraction?

- Page 29. Il est évoqué à plusieurs reprises des signaux de sécurité graves. Je m'autorise une réflexion sur un passé récent. J'étais président de la SOFFCO quand il a fallu porter à la HAS le dossier de la sleeve gastrectomie. Je recevais simultanément à l'HEGP chaque semaine des fistules après sleeve dont certaines ont mis plus d'un an à guérir, avec gastrectomies totales [11]. Cela ne m'a jamais conduit à adresser des signaux de sécurité graves à la HAS car je pense que la chirurgie de l'obésité est une nouvelle aventure, seul moyen actuel de guérison de l'obésité morbide et qu'il faut se laisser le temps d'optimiser les opérations, ce qui est en train d'être fait pour la sleeve gastrectomie mais ne pourra pas l'être pour le bypass en oméga si on l'interdit.
- Page 36. Les défauts du bypass en Y : à mon avis la morbidité de la deuxième anastomose est sous-estimée. Outre les hernies internes qui peuvent survenir n'importe quand, lors d'une grossesse, même de nombreuses années après le bypass, il a été démontré [12] que de nombreux patients se plaignaient de douleurs abdominales après bypass en $\mathrm{Y}$, mais pas après bypass en oméga.

- Page 62. Permettez-moi de réagir à cette phrase : «Certains experts expriment des réserves sur les résultats de la cohorte française de 2015 qui, de façon surprenante, ne rapporte que deux complications nutritionnelles alors que l'anse BP du BPGO était de $200 \mathrm{~cm} »$. Ceci concerne nos travaux, et je suis en droit de répondre à cette fausse affirmation.

Si l'OAGB est durablement déremboursé, il faudra que nous réalisions les PHRC nécessaires (avec dérivation d' $1,5 \mathrm{~m}$ ), mais que de temps perdu !

\section{Références}

1. Chevallier JM, Arman GA, Guenzi M et al. (2015) One Thousand Single Anastomosis (Omega Loop) Gastric Bypasses to treat morbid obesity in a 7-year period: Outcomes show few complications and good efficacy. Obes Surg; 25(6): 951-8.

2. Bruzzi M, Rau C, Chevallier JM (2014) Single anastomosis or mini-gastric bypass: long-term results and quality of life after a 5-year follow-up. Surg Obes Relat Dis. pii: S1550-7289(14)00341-4. doi: 10.1016/j.soard.2014.09.004

3. Guenzi M, Arman G, Chevallier JM (2015) Remission of type 2 diabetes after omega loop gastric bypass for morbid obesity. Surg Endosc 29(9): 2669-74

4. De Luca M, Tie T, Ooi G et al. (2018) Mini Gastric Bypass - One Anastomosis Gastric bypass (MGB-OAGB): IFSO Position Statement. Obes Surg; 28(5):1188-206

5. Ramos A, Chevallier JM, Mahawar K, et al. (2019) IFSO International Consensus Conference on One Anastomosis Gastric Bypass. A practical guide for OAGB-MGB. IFSO, Naples (Italy)/ Johnson and Johnson.

6. M Bruzzi, H Duboc, C Gronnier, JM Chevallier (2017) Long-term evaluation of biliary reflux after experimental One-anastomosis Gastric bypass in rats. Obes Surg 27(4):545-7

7. M Bruzzi, Thèse de Doctorat d'Université, Université de Paris, 15 novembre 2019. Evaluation du Risque de cancer oesogastrique sur reflux biliaire après bypass gastrique en omega.

8. Carbajo MA, Luque de Leon E, Jimenez JM et al. (2017) Laparoscopic One-Anastomosis Gastric Bypass: technique, results, and long-term Follow-up in 1200 patients. Obes Surg 27:1153-67

9. Kruschitz R, Luger M, Kienbacher C et al. (2016) The effect of Roux-en Y vs Omega loop gastric bypass on liver, metabolic parameters and weight Loss. Obes Surg 26(9): 2204-12

10. Parikh M, Eisenberg D, Johnson J et al. (2018) American Society for Metabolic and Bariatric Surgery: surgery review of the literature on One-anastomosis gastric bypass. Surg Obes Relat Dis. 14(8):1088-92

11. Moszkowicz D, Arienzo R, Khettab I, et al. (2013). Sleeve gastrectomy severe complications: is it always a reasonable surgical option? Obes Surg 23:676-86

12. Tartamella F, Ziccarelli A, Cecchini S et al. (2019) Abdominal pain and internal hernias after Roux-en-Y Gastric Bypass: are we dealing with the tip of an iceberg? Acta Biomed 90(2): 251-8 